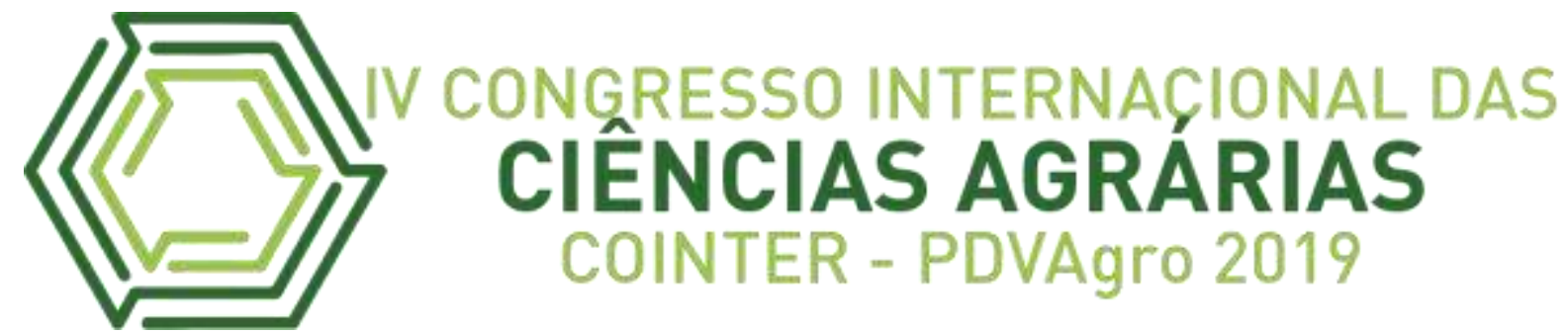

\title{
COOPERATIVISMO E O DESENVOLVIMENTO SOCIOECONÔMICO DA AGRICULTURA FAMILIAR NO BAIXO TOCANTINS, AMAZÔNIA BRASILEIRA
}

\section{COOPERATIVA Y EL DESARROLLO SOCIOECONÓMICO DE GRANJA FAMILIAR EN BAJO TOCANTINS, AMAZONIA BRASILEÑA}

\section{COOPERATIVE AND THE SOCIOECONOMIC DEVELOPMENT OF FAMILY FARM IN LOWER TOCANTINS, BRAZILIAN AMAZON}

Apresentação: Comunicação Oral

Raquel Lopes Nascimento ${ }^{1}$; Renan Yoshio Pantoja Kikuchi ${ }^{2}$; Andrey Rafael Moraes da Costa $^{3}$ Jorge Moura Serra Júnior ${ }^{4}$; Wagner Luiz Nascimento do Nascimento (Orientador) ${ }^{5}$

\section{DOI: https://doi.org/10.31692/2526-7701.IVCOINTERPDVAgro.2019.0167}

\begin{abstract}
Resumo
Este trabalho traz como foco discutir, no contexto da Amazônia, o cooperativismo como alternativa para o enfrentamento do desemprego, na busca de oportunidades de inclusão e estratégias de verticalização e dinamização de cadeias produtivas, no âmbito de desenvolvimento local ou territorial sustentável. O objetivo deste trabalho é traçar um perfil do cooperativismo na Região de Integração Tocantins "Baixo Tocantins", caracterizando a agricultura familiar dentro de uma perspectiva socioeconômica no contexto da Amazônia. Este trabalho faz parte do diagnóstico, realizado pela Incubadora Tecnológica de Desenvolvimento e Inovação de Cooperativas e Empreendimentos Solidários (INCUBITEC) do Instituto Federal de Educação, Ciência e Tecnologia do Pará - Campus Castanhal (IFPA - Campus Castanhal), com parceria do Sindicado e Organização das Cooperativas Brasileiras no Estado do Pará (OCB/PA). Foram aplicados questionário e consultado a análise estatística do Diagnóstico do Cooperativismo Paraense 2018, realizado pelo Sistema OCB/SESCOOP-PA (Serviço Nacional de Aprendizagem do Cooperativismo no Estado do Pará), assim como também foi realizada revisão bibliográfica. Os questionários foram aplicados no período de junho e julho de 2019, com cooperativas assistidas pela INCUBITEC e/ou ativas registradas no sistema OCB/PA. Sendo dirigidos pelos colaboradores e técnicos da incubadora, além de colaboradores da OCB/PA e estudantes do mestrado do IFPA Castanhal. Neste trabalho, foi possível concluir a elevada importância do cooperativismo no Baixo Tocantins, seguindo de uma perspectiva de sustentabilidade social, ambiental e econômica, além do fortalecimento das populações tradicionais que inclui os ribeirinhos, caboclos, índios e quilombolas. Como também contribuindo para o desenvolvimento da agricultura familiar e o extrativismo vegetal e valorização de produtos nativo da Amazônia, caracterizando a sociobiodiversidade.
\end{abstract}

\footnotetext{
${ }^{1}$ Agronomia, Instituto Federal de Educação, Ciência e Tecnologia do Pará, raquellopes.sdc@gmail.com

${ }^{2}$ Agronomia, Instituto Federal de Educação, Ciência e Tecnologia do Pará, renankukuchi18@gmail.com

${ }^{3}$ Agronomia, Instituto Federal de Educação, Ciência e Tecnologia do Pará, andreyrmcosta@gmail.com

${ }^{4}$ Administração, Serviço Nacional de Aprendizagem do Cooperativismo do Estado do Pará, SESCOOP/PA, Brasil, juniorserra@gmail.com

${ }^{5}$ Mestre, Instituto Federal de Educação, Ciência e Tecnologia do Pará, wagnerInnascimento@gmail.com
} 
Palavras-Chave: Cooperativismo, Amazônia, Agricultura Familiar, Extrativismo, Baixo Tocantins.

\section{Resumen}

Este trabajo aporta un enfoque para analizar, en el contexto de la Amazonía, las cooperativas como alternativa para afrontar el desempleo en busca de oportunidades para las estrategias de integración vertical y la inclusión y la promoción de cadenas productivas, en el contexto del desarrollo local y de los procesos territoriales sostenibles.El objetivo es elaborar un perfil cooperativo en el Tocantins "Inferior Tocantins" Integración Región con la agricultura familiar dentro de una perspectiva socio-económico en el contexto de la Amazonia. Este trabajo es parte del diagnóstico realizado por la Incubadora Tecnológica de Desarrollo y Cooperativas Innovación y Empresas de Solidaridad (INCUBITEC) del Instituto Federal de Educación, Ciencia y Tecnología de Pará - Campus Castanhal (IFPA - Campus Castanhal), en colaboración con el Sindicato y Organización las cooperativas brasileñas en el Estado de Pará (OCB / PA). cuestionario se aplica y se hace referencia a un análisis estadístico de diagnóstico Cooperative Paraense 2018, llevado a cabo por OCB System / SESCOOP-PA (Learning National Cooperative en el estado de Pará), y también se realizó revisión de la literatura. Los cuestionarios se aplicaron entre junio y julio de 2019, con la asistencia de las cooperativas INCUBITEC y / o activa registrada en el sistema OCB / PA. Son impulsados por los empleados y técnicos de la incubadora, así como los empleados de la OCB / PA y estudiantes de maestría IFPA Castanhal. En este trabajo, se concluyó la gran importancia de las cooperativas en el Bajo Tocantins, siguiendo el punto de vista de la sostenibilidad social, ambiental y económico, y el fortalecimiento de las poblaciones tradicionales, incluyendo riparia, mestizos, indios y cimarrones. Así como contribuir al desarrollo de la agricultura familiar y la extracción de la planta y la recuperación de los productos nativos del Amazonas, con el socio biodiversidad. así como los empleados de la OCB / PA y estudiantes de maestría IFPA Castanhal. En este trabajo, se concluyó la gran importancia de las cooperativas en el Bajo Tocantins, siguiendo el punto de vista de la sostenibilidad social, ambiental y económico, y el fortalecimiento de las poblaciones tradicionales, incluyendo riparia, mestizos, indios y cimarrones. Así como contribuir al desarrollo de la agricultura familiar y la extracción de la planta y la recuperación de los productos nativos del Amazonas, con el socio biodiversidad. así como los empleados de la OCB / PA y estudiantes de maestría IFPA Castanhal. En este trabajo, se concluyó la gran importancia de las cooperativas en el Bajo Tocantins, siguiendo el punto de vista de la sostenibilidad social, ambiental y económico, y el fortalecimiento de las poblaciones tradicionales, incluyendo riparia, mestizos, indios y cimarrones. Así como contribuir al desarrollo de la agricultura familiar y la extracción de la planta y la recuperación de los productos nativos del Amazonas, con el socio biodiversidad.

Palabras Clave: Cooperativismo, Amazon, la Agricultura Familiar, Extracción, Bajo Tocantins

\footnotetext{
Abstract

This work brings a focus to discuss, in the context of the Amazon, the cooperatives as alternative to face unemployment in search of opportunities for inclusion and vertical integration strategies and promotion of productive chains in the context of local development and sustainable territorial processes. The objective is to draw a cooperative profile in the
} 
Integration Region Tocantins "Lower Tocantins" featuring family farming within a socioeconomic perspective in the context of the Amazon. This work is part of the diagnosis made by the Technological Development Incubator and Innovation Cooperatives and Solidarity Enterprises (INCUBITEC) of the Federal Institute of Education, Science and Technology of Pará - Campus Castanhal (IFPA - Campus Castanhal), in partnership with the Trade Union and Organization Brazilian Cooperatives in the State of Pará (OCB / PA). questionnaire were applied and referred to statistical analysis Diagnostics Cooperative Paraense 2018, conducted by OCB System / SESCOOP-PA (National Cooperative Learning in the state of Pará), and was also performed literature review. The questionnaires were applied between June and July 2019, with cooperatives assisted by INCUBITEC and / or active registered in the OCB system / PA. They are driven by employees and technicians of the incubator, as well as employees of OCB / PA and students of master's IFPA Castanhal. In this work, it was concluded the high importance of cooperatives in the Lower Tocantins, following a perspective of social, environmental and economic sustainability, and the strengthening of traditional populations including riparian, mestizos, Indians and Maroons. As well as contributing to the development of family farming and plant extraction and recovery of native Amazon products, featuring the sociobiodiversity. as well as employees of OCB / PA and students of master's IFPA Castanhal. In this work, it was concluded the high importance of cooperatives in the Lower Tocantins, following a perspective of social, environmental and economic sustainability, and the strengthening of traditional populations including riparian, mestizos, Indians and Maroons. As well as contributing to the development of family farming and plant extraction and recovery of native Amazon products, featuring the sociobiodiversity. as well as employees of OCB / PA and students of master's IFPA Castanhal. In this work, it was concluded the high importance of cooperatives in the Lower Tocantins, following a perspective of social, environmental and economic sustainability, and the strengthening of traditional populations including riparian, mestizos, Indians and Maroons. As well as contributing to the development of family farming and plant extraction and recovery of native Amazon products, featuring the sociobiodiversity.

Keywords: Cooperativism, Amazon, Family Agriculture, Extraction, Lower Tocantins.

\section{Introdução:}

Segundo Chaddad e Cook (2004), a cooperativa é uma organização criada por um conjunto de indivíduos que compartilham algum interesse comum e percebem que, por meio de uma organização formal, esse objetivo comum é mais facilmente atingido. O cooperativismo está inserido na economia solidaria que tem crescido no Brasil como movimento alternativo de inserção de milhares de trabalhadores e trabalhadoras no mundo do trabalho.

Para Singer (1999) a Economia Solidária tem sido uma resposta importante dos trabalhadores e trabalhadoras em relação às transformações ocorridas no mundo do trabalho. Segundo o autor, são milhares de organizações coletivas, organizadas sob a forma de autogestão que realizam atividades de produção de bens e de serviços, crédito e finanças solidárias, trocas, comércio e consumo solidário tanto em áreas urbanas como rurais. Trata-se, pois de uma importante alternativa de inclusão social pela via do trabalho e da renda, combinando a 
cooperação, a autogestão e a solidariedade na realização de atividades econômicas (SINGER, 1999).

Segundo o Sistema de Organização das Cooperativas Brasileiras (OCB), (2019), o número de cooperativas no Brasil só vem aumentando ao longo do tempo, sendo que no ano de 2018 chegou a de 6.828 cooperativas, apresentando significativo ganho para a economia no país. Destas, 1.613 são do ramo agropecuário, totalizando 1.021 .019 cooperados e gerando um total de 209.778 empregos, tal que, o ramo agropecuário é o primeiro no ranking de quantidade de cooperativas, e geração de emprego.

A economia solidária torna-se alternativa para o enfrentamento do desemprego, na busca de oportunidades de inclusão e estratégias de verticalização e dinamização de cadeias produtivas no âmbito de processos de desenvolvimento local ou territorial sustentável.

No Pará, a agropecuária é de grande importância com crescimento significativo na economia do estado, principalmente com a agricultura familiar e o extrativismo e tem no cooperativismo uma vital ferramenta de desenvolvimento do setor OCB/PA, (2018).

Para agricultores, as cooperativas serviram décadas atrás como instrumentos coletivos de desenvolvimento econômico e de fortalecimento comunitário, mantendo-se acessíveis e próximas aos seus interesses Gaiger (2013). No contexto da Amazônia, há necessidade de se pensar desenvolvimento rural sustentável, levando em consideração sua diversidade de modos de produção e produtos, desenvolvidos ou originados por populações de ribeirinhos, caboclos, índios e quilombolas que se consideram agricultores, extrativistas, pescadores, artesões e etc.

Dessa forma, pretende-se traçar um perfil do cooperativismo na Região de Integração Tocantins "Baixo Tocantins", caracterizando a agricultura familiar dentro de uma perspectiva socioeconômica no contexto da Amazônia.

\section{Fundamentação Teórica}

\section{Concepção de divisão de espaço geográfico no Pará:}

O Estado do Pará representa 29,73\% da Amazônia brasileira e 14,65\% do território nacional. Dentro dessa unidade da federação estão grandes mesorregiões que foram determinadas a partir da Resolução da Presidência do IBGE nº ${ }^{\circ}$ 11, de 5 de junho de 1990, e são definidos como conjuntos de munícipios contíguos e que inseridos dentro de três dimensões: o processo social, como determinante, o quadro natural, como condicionante, e a rede de comunicação e de lugares, como elemento de articulação espacial.

Estas dimensões possibilita uma identidade regional dentro do espaço delimitado e, mais 
ainda, quando se trata das microrregiões, também instituída pela Resolução da Presidência do IBGE $\mathrm{n}^{\mathrm{o}}$. 11, de 5 de junho de 1990 e que foram definidas como partes das mesorregiões que apresentam especificidades, quanto à organização do espaço.

Segundo a Resolução da Presidência do IBGE, esta organização está relacionada com estruturas de produção diferenciadas e relações de vida ao nível local, em outras palavras é constitui-se pela produção propriamente dita, distribuição, troca e consumo, incluindo atividades urbanas e rurais.

Além das mesorregiões e microrregiões estabelecidas, em 2007 passou-se a utilizar, no Pará, o conceito de Região de Integração (RI) no âmbito da criação da Secretaria de Estado de Integração Regional (Seir) Oliveira et al, (2011). Dessa forma, obtendo um espaço mais homogêneo e de identidade peculiar deste espaço geográfico.

O Pará está dividido em 6 mesorregiões: Baixo Amazonas Paraense, Marajó, Metropolitana de Belém, Nordeste Paraense, Sudoeste Paraense e Sudeste Paraense. E nas mesorregiões estão inseridas as microrregiões que no total são 22. As Regiões de Integração (RI), segundo a SEPLAN (Secretaria de planejamento do Pará), são no total de 12.

O Região de Integração Tocantins, é composto por 11 munícipios: Abaetetuba, Acará, Baião, Barcarena, Cametá, Igarapé-Miri, Limoeiro do Ajuru, Mocajuba, Moju, Oeiras do Pará e Tailândia. Estes munícipios integram três microrregiões do Estado do Pará: a microrregião de Cametá, de Belém e Tomé-Açu (Figura 1).

Apesar de Região de Integração ser apresentado, com seu surgimento a partir do ano de 2007 (OLIVEIRA ET AL, 2011; BASTOS ET AL, 2010), bem antes de disso, no Plano Territorial De Desenvolvimento Rural Sustentável Do Baixo Tocantins para o ano de 2011 a 2014, é referido que esta região já havia se autodenominado, pelos próprios atores locais, como "Baixo-Tocantins", tal que é o termo mais utilizado para denominar a Região de Integração Tocantins.

\section{Território e Territorialidade no contexto da Amazônia brasileira}

A estratégia de regionalização, através de várias subdivisões de espaço geográfico na Amazônia, demonstra uma complexa diversidade ambiental, cultural, social e política econômica que existe no espaço.

No trabalho de Schneider e Tartaruga, 2006 na perspectiva de território, afirma que o desenvolvimento pressupõe a ação sobre o espaço e a mudança das relações sociais nele existente. Da mesma forma, Mariani et al, (2010), também traz uma abordagem nesse sentido, 
tal que afirma ser necessário para explicar as dinâmicas de desenvolvimento tendo como pressuposto o espaço social e as relações de poder nele operantes.

Entender os fatores que constituem esta abordagem territorial do desenvolvimento requer analisar o papel dos múltiplos agentes, das instituições, das demais formas organizativas da sociedade e, até mesmo, da cultura na configuração do espaço social (SCHNEIDER, 2004).

Para a Amazônia, estes aspectos são muito incisivos, pois a diversidade de atividades praticadas dentro da agricultura familiar e as diferentes formas organizacionais desta categoria possuem uma relação com os territórios bastante imponente que os diferem entre si dentro dos estados, mesorregiões e até mesmo nas microrregiões.

Aspecto de diversidade e complexidade do espaço influencia a interação do homem com a natureza conferindo peculiaridades como abordados por Mendes, (2010) que trata sobre a interferência do homem na natureza e suas transformações no modo de vida das populações.

A compreensão da espacialidade é fundamental para concepção da diversidade socioeconômica. Segundo Schneider et al, (2014) é um avanço o reconhecimento da definição e compreensão das características e do significado do grupo social denominado agricultura familiar.

Schneider et al, (2014) considera o principal avanço - no que diz respeito à melhor definição da agricultura familiar - o reconhecimento da enorme diversidade econômica e heterogeneidade social, deste grupo que trabalha mediante o uso da força de trabalho dos membros de suas famílias e que produzem tanto para seu autoconsumo como para a comercialização.

\section{Caracterização de áreas de várzeas no baixo Tocantins}

O Baixo Tocantins é uma região que sofre influência de marés, sendo esta, uma área de várzea tradicionalmente ribeirinha que nada mais é do que a população que habita às margens dos rios e igarapés, com características típicas da Amazônia, tal que, para Chaves (2001), os agentes sociais identificados como ribeirinhos, vivem em agrupamentos comunitários com várias famílias, localizados, como o próprio termo sugere, ao longo dos rios e seus tributários (lagos). As populações ribeirinhas do Baixo Tocantins são caracterizadas também pela dinâmica extrativista vegetal e animal, como a pesca, coleta de açaí (Euterpe oleracea Mart.), andiroba Carapa guianensis A.), cacau (Passiflora cacao) e etc.

Estes concernem características peculiares na formação econômica, social e política da Amazônia, são populações que segundo Lira et al (2016), detém de um amplo saber sobre o 
ambiente amazônico e suas diversas formas de uso e manejo, apropriando-se de recursos florestais, baseado na reciprocidade com a natureza percebendo o tempo ecológico dos recursos naturais para organizar o trabalho na heterogeneidade das diversas formas de utilização dos recursos naturais.

Nisto, Lira et al, (2016) afirma que há uma relação de respeito entre homem-natureza, tendo em vista que os ribeirinhos não dissociam o homem da natureza, permitindo o manejo do ambiente sem a degradação dos recursos naturais, a partir do etnoconheciemento.

O Baixo Tocantins é organizado pelos rios Moju, Acará, Pará e o grandioso Tocantins que deu o nome a região. Os rios da região são utilizados como vias para transporte de cargas e pessoas (Figura 1). O conjunto de rios, furos e igarapés desta região é plenamente navegável, durante todo o ano, o que permite um fluxo intenso de embarcações dos mais variados calados e capacidades de operação.

O solo da região é formado por terra firme e várzea, o solo de várzea está localizado nas margens dos rios, sendo adubados e drenados naturalmente pelas enchentes periódicas. São ricos em húmus (matéria orgânica), que são depositadas as margens dos rios

\section{O Cooperativismo como forma de desenvolvimento rural sustentável}

Araújo et. al. (2007), ressaltam que os princípios norteadores da transformação da agricultura familiar se alinham ao associativismo e à participação social.

O cooperativismo vem no sentido de melhorias de produção, escoamento, verticalização, beneficiamento, comercialização, geração de renda e trabalho que consequentemente, atua na melhoria da condição de vida, fortalecimento e desenvolvimento de populações tradicionais.

As cooperativas buscam realizar funções econômicas, através de uma empresa, mas vinculadas ao social, pois buscam prestar serviços que visem satisfazer necessidades das pessoas Schneider (2006). Dessa forma, as cooperativas estão inseridas dentro do que chamamos de economia solidária.

Para Reis et al, (2015) a economia solidária é uma forma de resistência aos efeitos da exploração capitalista do trabalho, tal que a economia capitalista tem gerado desigualdades socais e regionais como consequência da divisão internacional do trabalho, dessa forma, a economia solidária torna-se uma alternativa.

Como afirmam GOERCK (2005) e SOUSA; BARBOSA; REIS (2009), a economia solidária é uma estratégia que permite a dinamização socioeconômica no âmbito de processos de desenvolvimento local e/ou territorial sustentável, promovendo a coesão social, a 
preservação da diversidade cultural e o meio ambiente.

\section{Metodologia}

A área de abrangência do Baixo Tocantins é de 36.024,20 Km², sendo composta por 11 municípios. A cobertura vegetal na região do Baixo Tocantins é formada de floresta Ombrófila Densa, com áreas de matas, de várzeas e de igapós.

O Território do Baixo Tocantins encontra-se numa zona de fronteira, está localizado entre a Amazônia Central e Amazônia Oriental, no nordeste do Pará, por onde passa a microrregião da Bacia do rio Tocantins, considerada a segunda mais importante do país, superada apenas pela Bacia do Rio Amazonas. O rio Tocantins, faz parte do complexo estuário amazônico, se comunicando com o rio Pará e o rio Guamá, que se juntam na foz do rio Amazonas.

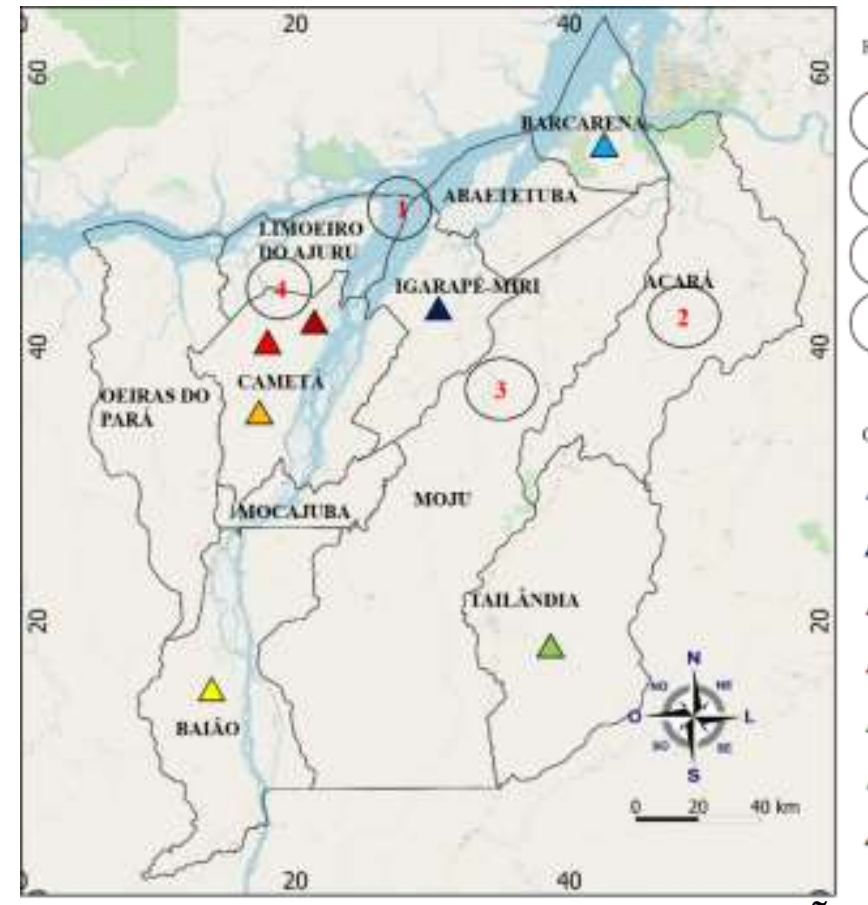

ROOS DO BAXXO TOCANTINS

(1) TocaNtins-Foz
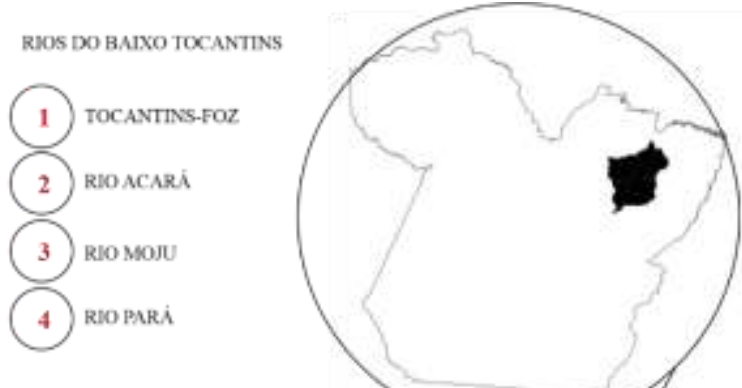

COOPERATIVAS DO BAIXO TOCANTINS

$\triangle$ CEDAB

A CAEPM

$\Delta$ CART

$\triangle$ COOPEMUC

$\triangle$ COOPRUBAI

$\triangle$ CART

$\triangle$ COOAPEBAL

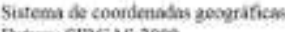
Dudum SIRCiAs 3000

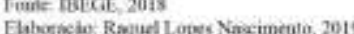

FIGURA 1: MAPA DE LOCALIZAÇÃ̃O DA REGIÃO DE INTEGRAÇÃO TOCANTINS (BAIXO TOCANTINS)

Fonte: Autores

Este trabalho faz parte do diagnóstico, realizado pela Incubadora Tecnológica de Desenvolvimento e Inovação de Cooperativas e Empreendimentos Solidários (INCUBITEC) do Instituto Federal de Educação, Ciência e Tecnologia do Pará - Campus Castanhal (IFPA - 
Campus Castanhal), com parceria do Sindicado e Organização das Cooperativas Brasileiras no Estado do Pará (OCB/PA), sendo esta, entidade de representação das necessidades dos cooperados. Os questionários foram aplicados no período de junho e julho de 2019, com cooperativas assistidas pela INCUBITEC e/ou ativas registradas no sistema OCB/PA. Sendo dirigidos pelos colaboradores e técnicos da incubadora, além de colaboradores da OCB/PA e estudantes do mestrado do IFPA Castanhal.

A realização deste trabalho se deu através do levantamento de dados por meio questionário junto as cooperativas a região do Baixo Tocantins. Foram consultadas 5 cooperativas das 7 que são assistidas pela INCUBITEC e/ou ativas no sistema OCB/PA (Tabela 1). As cooperativas estudadas são: CEDAB (Barcarena); CART, COOPEMUC (Cametá); CAEPIM (Igarapé-Miri) e CART (Tailândia) (Figura 1).

\section{TABELA 1 COOPERATIVAS AGRÍCOLAS POR MUNÍCIPIO DO BAIXO TOCANTINS}

\begin{tabular}{c|cc}
\multicolumn{1}{c}{ MUNÍCIPIO } & \multicolumn{1}{c}{ SIGLAS } & NOME \\
\hline BAIÃO & COAPEBAL & Cooperativa Mista Agropecuária De Baião Ltda \\
BARCARENA & CEDAB - & Cooperativa De Extração E Desenvolvimento Agrícola De Barcarena \\
CAMETÁ & COOPRUBAT & Cooperativa Dos Produtores Rurais Do Baixo Tocantins \\
CAMETÁ & CART & Cooperativa Agroextrativista Resistência de Cametá \\
CAMETÁ & COOPEMUC & Cooperativa Agroindustrial de Mulheres do Município de Cametá \\
IGARAPÉ-MIRI & CAEPIM & Cooperativa Agrícola de Empreendedores Populares de Igarapé-Miri \\
TAILÂNDIA & CART & Cooperativa Dos Agricultores Da Região De Tailândia
\end{tabular}

Fonte: Autores

Além dos dados de questionário, foi utilizado análise estatística do Diagnóstico do Cooperativismo Paraense 2018, realizado pelo Sistema OCB/SESCOOP-PA (Serviço Nacional de Aprendizagem do Cooperativismo no Estado do Pará), assim como revisão bibliográfica.

A formulação e validação dos questionários, concretizou-se por meio de reuniões, mais precisamente em workshop de cooperativismo - realizado pela INCUBITEC, onde foram estabelecidas, junto a OCB/PA, as questões, mas pertinentes, a cerca de: identificação e organização social, verticalização de produção, atividades econômicas, gestão, comercialização e questões voltadas para o meio ambiente e sustentabilidade.

Para a análise dos dados obtidos dos questionários, foi realizada a técnica de estatística descritiva e assim, estabelecendo a caracterização da área de estudo, junto as reflexões da fundamentação teórica, discutidas neste artigo. 


\section{Resultados e Discussão:}

O cooperativismo agrícola no Pará é bem expressivo em número, tal que, segundo o Diagnóstico do Cooperativismo Paraense 2018 (OCB/SESCOOP-PA, 2019), as cooperativas agrícolas estão em segundo lugar no ranking em quantidade de cooperativas no estado do Pará, estando atrás apenas do ramo de transporte. O total de cooperativas é 215, com 12 ramos de atuação, destas, $29 \%$ são cooperativas agropecuária o que significa, 62 cooperativas que atuam para o fortalecimento de produção agrícola (Gráfico 1).

\section{GRÁFICO 1: RAMO DE ATUAÇÃO DE COOPERATIVAS, POR QUANTIDADE E GERAÇÃO DE EMPREGO.}

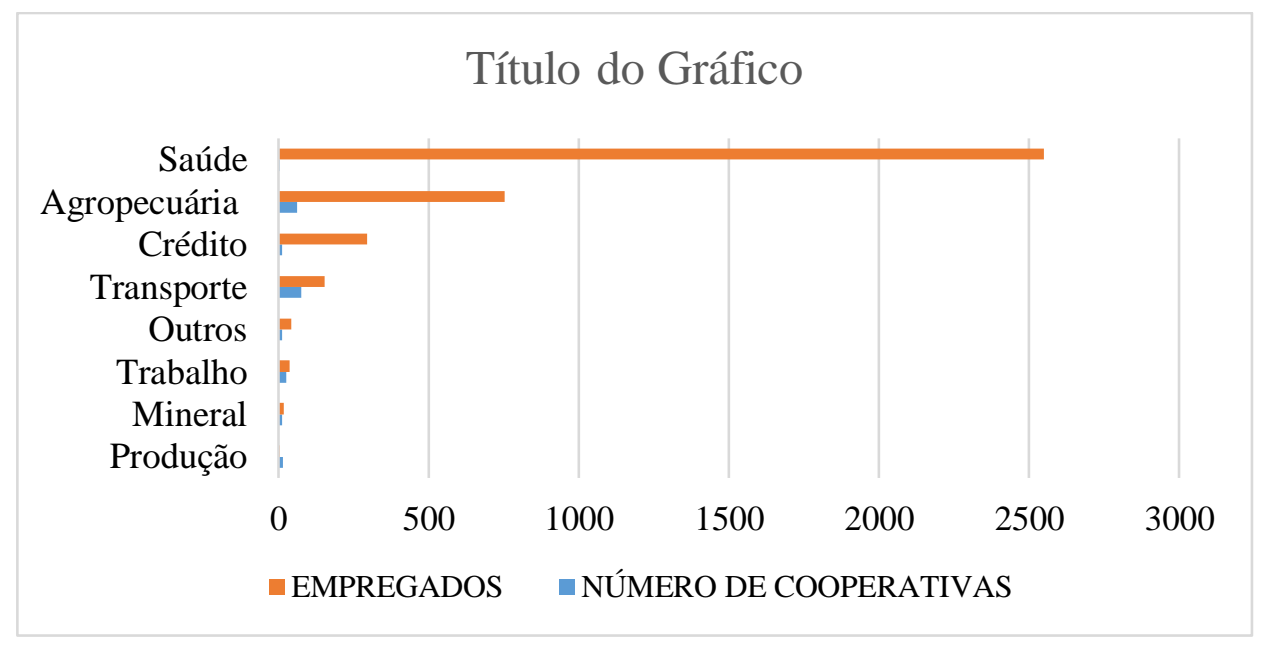

Fonte: Adaptado de OCB/PA, 2019

Em número de cooperados, as cooperativas agrícolas, estão em terceiro lugar com 6.083, estando atrás do ramo mineral com 47.281 cooperados e o ramo de crédito, com 30.136. Contudo, o número de empregados, é bem mais significativo, onde são gerados 753 empregos, apenas em cooperativas agrícolas, sendo a quantidade mais expressiva, depois do ramo de saúde (Gráfico 1).

As cooperativas apresentam um impacto socioeconômico no meio rural, resultante da organização dos agricultores familiares na busca de melhorias na qualidade de vida, assim, como afirma Petrella (2007), que as cooperativas exercem de forma interna e externa uma influência socioeconômica sobre a sociedade a fim de melhorar as condições de vida humana.

Neste sentido, compartilhando da ideia de Reis et al (2015) e Laville et al, (2004), a organização social cria um espaço estratégico para o enfrentamento dos efeitos de sistema capitalista, garantindo-lhes acesso a direitos sociais, de forma que se construa uma sociedade mais justa. 
Na pesquisa realizada, verificou-se que a principal finalidade de se construir uma cooperativa, está baseada em: organização dos produtores rurais para a autonomia e independência; melhorar a situação econômica de seus cooperados; viabilizar a comercialização dos produtos. Além destes, foram pontuados como importantes a geração e/ou a melhoria de renda dos sócios e a eliminação de intermediários para a comercialização de seus produtos.

Dessa forma, a organização da comunidade se torna alternativa para superação de desafios e dificuldades encontrados na dinâmica da cadeia produtiva, seja originado do cultivo ou de extração vegetal e/ou animal. Quando se trata da Amazônia, o perfil de produtos e produção agrícola é difícil de definir, pois apresenta uma série de peculiaridades e diversidades que são distintas, mesmo dentro do contexto de bioma amazônico.

Neste sentido, a maioria dos cooperados, quase que predominantemente são extrativistas; ribeirinhos e autoconsideram enquadrados na categoria de agricultores familiares, mas além destes, tem também indígenas, quilombolas e pescadores artesanais. Mesmo dentro uma região, que corresponde a aproximadamente 3\% do território paraense, o Baixo Tocantins imensamente diverso, na sua população, produção, produtos agrícolas e extrativistas.

As diferentes formas de produção agrícola desenvolvidas por populações locais como os ribeirinhos, caboclos, índios e quilombolas, por exemplo; sempre estiveram presentes na construção da identidade amazônica e perduram até os dias atuais. Suas diversas atividades lhes conferem características próprias que necessitam ser conhecidas e colocadas em pauta na construção de políticas públicas coerentes com as especificidades locais.

Para Hurtienne, (2005), o desafio para a análise da dinâmica de produção familiar na Amazônia, reside na dificuldade de considerar a grande diversidade das formas de produção familiar no campo que estão relacionados a:

I- Extrativistas tradicionais e agricultores itinerantes, como os grupos indígenas, caboclos e ribeirinhos, foram os grupos populacionais mais importantes na Amazônia rural - o segmento desses camponeses agroextrativistas ainda é importante na Zona Bragantina;

II- Com a construção da ferrovia na Zona Bragantina no começo do século, estabelece-se um campesinato agrícola com base numa agricultura itinerante de pousio, já altamente orientado para o suprimento do mercado de Belém nos anos 40 e 50, esse processo de colonização estendeu-se à região Guajarina (Capitão Poço e Irituia);

III- A grande imigração de colonos do Nordeste e do Sul do Brasil depois da abertura da Amazônia através dos novos eixos rodoviários, os programas de 
colonização oficial e os grandes projetos foram a base para a formação de um campesinato mais novo.

Segundo Schröder (2010) não dá para simplificar a estrutura social e produtiva do meio rural amazônico. Neste mesmo sentido Hurtienne, (2005) afirma que não há uma linha divisória para determinar o modo de produção familiar.

Aspecto de diversidade e complexidade da Amazônia são fundamentais para entender a forma de organização e relação do homem com a natureza, como bem abordado por Schneider, (2004) que levanta uma discussão de pluriatividade no âmbito da agricultura familiar que muito bem cabe ao contexto da Amazônia.

A pluriatividade é um fenômeno heterogêneo e diversificado que está ligado, de um lado, às estratégias sociais e produtivas que forem adotadas pela família e por seus membros e, de outro, dependerá das características do contexto em que estiver inseridos Schneider, (2004).

A diversidade de atividades econômicas praticadas dentro da agricultura familiar e as diferentes formas organizacionais desta categoria possuem uma relação com os territórios bastante imponente que os diferem entre si dentro de um mesmo espaço e isso se reflete na variedade produtos agrícolas gerados nas cooperativas.

A atividade econômica da cooperativa está atrelada justamente como os sócios se autoidentificam e a região que estão inseridos, sendo que o produto mais significativo, dentro dos empreendimentos é o açaí (Euterpe oleracea) (Gráfico 2). Segundo o levantamento do IBGE, 2018, o açaí é principal produto da extração vegetal, no estado do Pará, o que também é confirmado pela atividade realizadas pelas cooperativas estudas na região do Baixo Tocantins. 


\section{GRÁFICO 2: PRINCIPAIS PRODUTOS PRODUZIDOS E/OU COMERCIALIZADOS DE ORIGEM AGRÍCOLA E EXTATIVISTA.}

A maioria dos produtos são nativos e seguem um modelo de produção agroecológico e orgânico, o que demonstra a valorização e fortalecimento da sustentabilidade social, ambiental e econômico de uma região que é tão diversa de fauna, flora, cultura e modelos de produção.

Seguindo da mesma perspectiva de pesquisadores brasileiros, como Maluf et al. (2015); Burlandy et al. (2014) e Castro, (2015), que apontam os modelos agroecológico de produção e de processamento de alimentos, como alternativa para o enfrentamento de questões nutricionais, sociais e ambientais decorrentes do sistema agroalimentar hegemônico, a produção agrícola realizada por essas cooperativas, não está firmada apenas aos benefícios econômicos, mas também ao social e ambiental, fortalecendo o desenvolvimento local sustentável.

A diversidade de produtos produzidos e/ou comercializados pelas cooperativas da

\section{Fonte: Autores}

Região do Baixo Tocantins são de origem, principalmente, do extrativismo e nesse sentindo é importante ressaltar o debate em torno do termo "produtos da sociobiodiversidade" que está relacionado justamente com a atividade extrativista. Dessa forma, a relevância social, ambiental e econômico do extrativismo, especialmente de populações ribeirinhas, apresenta uma relação recíproca de troca do homem com a biodiversidade, no qual se concerne a necessidade de manutenção de ambos, tanto para a garantia de produção futura, quanto para o protagonismo das populações e comunidades tradicionais que se beneficiam dos produtos da

\section{PRINCIPAIS PRODUTOS AGRÍCOLAS E EXTRATIVISTAS}

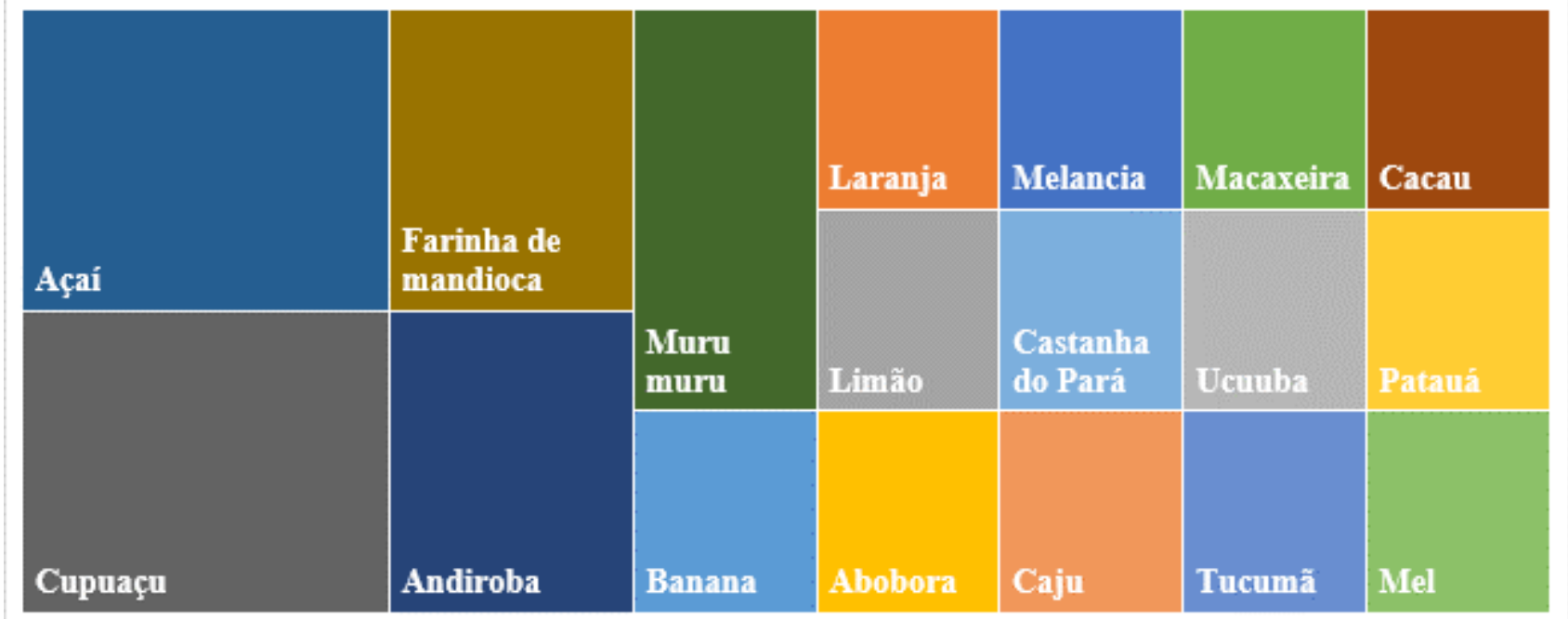

sociobiodiversidade. 
Os produtos da sociobiodiverdade para as populações tradicionais são de grande relevância e expressam a identidade territorial e social da região e ainda contribui para a economia.

\section{Conclusões}

Com esta pesquisa foi possível concluir a elevada valia do cooperativismo agrícola, no âmbito da Amazônia brasileira como forma de desenvolvimento rural sustentável de populações tradicionais que inclui os ribeirinhos, caboclos, índios e quilombolas. Dessa forma, além do fortalecimento destas populações, há também a valorização da diversidade e peculiaridades do território amazônico.

O cooperativismo apresenta significativa importância para o desenvolvimento e fortalecimento da agricultura familiar e extrativismo na região do Baixo Tocantins, no qual podemos visualizar, através dos números a relevância da organização social frente a sociedade que valoriza o individualismo e a divisão.

Foi possível observar a diversidade de formas de produção e dos produtos advindos da agricultura e do extrativismo vegetal, caracterizando a sociobiodiversidade realizado pelas cooperativas do Baixo Tocantins. Por fim, o cooperativismo, é de elevado relevância para o fomento da sustentabilidade social, ambiental e econômica na Amazônia.

\section{Referências}

ARAÚJO, T. C. A. de; GODRIM, M. D.; SOUZA, V. S. A Organização Social da Agricultura Familiar do Projeto Jaíba- MG, como desafio para o desenvolvimento local sustentável. EMATER-MG, JAIBA, BRASIL. 2007. Disponível em: $<$ http://www.sober.org.br/palestra/6/333.pdf> Acesso em: 10 de out. de 2019.

BASTOS, A. P. V.; ALMEIDA, O.; CASTRO, E. R; MARIN, R. A.; PIMENTEL, M. S.; RIVERO, S; SILVA, I. C; TORRES, I.; BRÜZEKE, F.J. Economia E Sociedade Na Região Do Tocantins, Pará. PAPERS DO NAEA Nº 259, UFPA. ISSN 15169111. Belém, março de 2010 .

BURLANDY, L.; GOMES, F.S.; CARVALHO, C.M.P.; DIAS, P.C.; HENRIQUES, P. 2014. Intersetorialidade e potenciais conflitos de interesse entre governos e setor privado comercial no âmbito das ações de alimentação e nutrição para o enfrentamento de doenças crônicas não transmissíveis. Visa em debate 2(4):124-29. 
CASTRO, I.R.R. Desafios e perspectivas para a promoção da alimentação adequada e saudável no Brasil. Cadernos de Saúde Pública 31(1):7-9. 2015

COOK, M. L., CHADDAD, F. R. “Advances in Cooperative Theory since 1990: A Review of Agricultural Economics Literature." In G.W.J. Hendrikse (ed.), Restructuring Agricultural Cooperatives, pp. 65-90, Haveka, the Netherlands. 2004

CHAVES, Maria P. S. R. Uma experiência de pesquisa -ação para gestão comunitária de tecnologias apropriadas na Amazônia: o estudo de caso do assentamento de Reforma Agrária Iporá. 2001. Tese (Doutorado em Política Científica e Tecnológica) Universidade Estadual de Campinas, Campinas, SP. 2001

CORDEIRO, M. C.C.; VASCONCELOS, L.G. T.; SCHWARTZ G.; OLIVEIRA, F.A. Nordeste Paraense: panorama geral e uso sustentável das florestas secundárias. Belém: EDUFRA, 2017.

DIAS, Guilherme A. V. OLIVEIRA, Wesley. Região De Integração Tocantins-Pa: Um Novo Olhar Sobre O Extrativismo E Alguns Aspectos Socioeconômicos. Texto de discussão. IPEA - Instituto de pesquisa Econômica Aplicada. Rio de Janeiro, março de 2011

GAIGER, L. I. A Economia Solidária e a Revitalização do Paradigma Cooperativo. Revista Brasileira de Ciências Sociais. Vol. 28, $\mathrm{N}^{\mathrm{o}}$ 82, Jun/2013. Disponível em: http://www.scielo.br/pdf/rbcsoc/v28n82/v28n82a13.pdf.

GAIGER, L. I. O Mapeamento Nacional e o Conhecimento da Economia Solidária. Revista da ABET. Vol. 12, No 1, Jan/Jun 2013. ISSN 1679-2483. Disponível em: http://periodicos.ufpb.br/ojs/index.php/abet/issue/view/1379/showToc.

GOERCK, Caroline. Economia popular solidária: no processo de reestruturação produtiva Brasileira. Revista Virtual Textos \& Contextos, $n^{\circ}$ 4, dez. 2005.

HURTIENNE, T. Agricultura familiar e desenvolvimento rural sustentável na Amazônia. Novos Cadernos NAEA. v. 8, n. 1 - p. 019-071 jun. 2005 
Brasil em mesorregiões e microrregiões geográficas. 1990.

INSTITUTO BRASILEIRO DE GEOGRAFIA E ESTATÍSTICA-IBGE. LSPA Levantamento Sistemático da Produção Agrícola 2018 . Disponível em: https://www.ibge.gov.br/estatisticas/economicas/agricultura-e-pecuaria.html.

Acesso em: 10 de out de 2019.

INSTITUTO BRASILEIRO DE GEOGRAFIA. DEPARTAMENTO DE GEOGRAFIA. Divisão do Brasil em micro-regiões homogêneas: 1968. Edicao do Departamento de Documentacao e Divulgacao Geografica e Cartografica, 1970.

LAVILLE, J. L. e FRANÇA FILHO, G. C. Economia Solidária, uma abordagem internacional. Porto Alegre: Editora da UFRGS, 2004.

Lima, Maria Helena Palmer et al. Divisão Territorial Brasileira. Fundação Instituto Brasileiro De Geografia E Estatística - Departamento de Geografia, 2002

LIRA, T. M.; CHAVES, M. P. S. R. Comunidades ribeirinhas na Amazônia: organização sociocultural e política. Campo Grande, MS, v. 17, n. 1, p. 66-76, jan./mar. 2016.

LOUREIRO, V. R. Amazônia: Uma história de perdas e danos, um futuro a (re)construir. Estudos Avançados. Vol. 16, $\mathrm{N}^{\mathrm{o}}$ 45, pag. 107-121. 2002. Disponível em: http://www.scielo.br/pdf/ea/v16n45/v16n45a08.pdf.

MALUF, R.; BURLANDY, L.; SANTARELLI, M.; SCHOTTZ, V.; SPERANZA, J.S. Agricultura sensível à nutrição e a promoção da soberania e da segurança alimentar e nutricional no Brasil. Ciência e Saúde Coletiva 20(8):2303-2312. 2015

MARIANI, M. A. P.; ARRUDA, D. O. Território, Territorialidade E Desenvolvimento Local: Um Estudo De Caso Dos Empreendimentos Econômicos Solidários De Corumbá/Ms. SOBER - Sociedade Brasileira de Economia, Administração e Sociologia Rural. Campo Grande, 25 a 28 de julho de 2010

MDA _ Ministério de Desenvolvimento Agrário. Plano Territorial De Desenvolvimento Rural Sustentável Do Baixo Tocantins. 2010 
OCB - Sistema de Organização das Cooperativas Brasileiras. (2019). Números Do Cooperativismo brasileiro. Disponível em: https://www.ocb.org.br/numeros. Acesso em: 10 de out de 2019

OCB/PA - Sistema de Organização das Cooperativas Brasileiras. Diagnóstico e censo do Cooperativismo Paraense 2018.

PETRELLA, R. Pour une nouvelle narration du monde. Montréal: Éditions Écosociété, 2007.

REIS, Adebaro Alves et al. Agricultura Familiar e Economia Solidária: a experiência da Associação MUTIRÃO, na região do Baixo Tocantins, Amazônia Paraense. Revista Tecnologia e Sociedade, v. 11, n. 22, p. 120-142, 2015.

SANTOS, Amanda Rayana et al. Pluriatividade como estratégia de renda: o caso de um agricultor familiar na comunidade ribeirinha São João Batista, Pará. Revista Tecnologia e Sociedade, v. 11, n. 23, p. 89-105, 2015.

SCHNEIDER, S.; CASSOL, A. Diversidade E Heterogeneidade Da Agricultura Familiar No Brasil E Algumas Implicações Para Políticas Públicas. Cadernos de Ciência \& Tecnologia, Brasília, v. 31, n. 2, p. 227-263, maio/ago. 2014

SCHNEIDER, Sergio et al. A abordagem territorial do desenvolvimento rural e suas articulações externas. Sociologias, v. 6, n. 11, 2004.

SCHNEIDER, Sergio. Território, ruralidade e desenvolvimento. Org.). Las Configuraciones de los Territorios Rurales en el Siglo XXI, v. 1, p. 67-108, 2009.

SINGER, P. Globalização e Desemprego: diagnóstico e alternativas. São Paulo: Contexto, 1998

SINGER, Paul. A crise das relações de trabalho. Relações de trabalho contemporâneas. Belo Horizonte: IRT (Instituto de Relações do Trabalho) da Pontifícia Universidade Católica de Minas Gerais, p. 31-45, 1999.

SEPLAN-Secretaria de Planejamento do Pará. Perfil de RI Tocantins. Disponível em: 
http://seplan.pa.gov.br/regi\%C3\%B5es-de-integra\%C3\%A7\%C3\%A3o. Acesso em: 05 de out de 2019

SOUZA, A. L.; BARBOSA, M. J. de S.; REIS, A. A. dos. Incubadoras universitárias: inovação social e desenvolvimento. In. Tecnologia social, economia solidária e políticas públicas. BOCAYUVA, P. C. C.; VARANDA, A. P. de M. (org.). ed. 1. Rio de Janeiro: FASE: IPPUR, UFRJ, 2009.

Uma outra economia é possível: Paul Singer e a Economia Solidária. André Ricardo de Souza, Gabriela Cavalcanti Cunha, Regina Yoneko Dakuzaku (orgs). São Paulo. Contexto, 2003. 